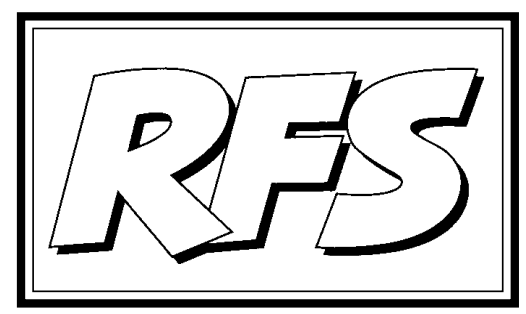

Revista de Fomento Social, 56 (2001), 715-728

\title{
La integración y el desarrollo de Centroamérica en el siglo XXI: Una conferencia regional ${ }^{1}$
}

El pasado día 27 de julio de 2001 se celebró en Tegucigalpa (Honduras) la conferencia regional ${ }^{3}$ sobre la integración y el desarrollo de Centroamérica en el siglo XXI que celebraba el 40 aniversario de la constitución del Banco Centroamericano de Integración Económica (BCIE). El acto fue inaugurado con las palabras del Presidente del BCIE, Pablo Schneider.

1 Esta crónica debió aparecer en el número anterior de la Revista, habíendose quedado fuera por un error de composición del mismo. A ella se hacía alusión precisamente en la primera nota a pie de página del artículo de Gert ROSENTHAI, p. 461.

2 Profesor de ETEA.

3 El término regional en América latina tiene una acepción diferente a la común en el entorno europeo. En todo el texto se debe entender región como grupo de países de América Latina que participan en algún tipo de acuerdo de integración. 
La conferencia celebraba este destacado momento del proceso centroamericano de integración en un período de recuperación de las expectativas regionales sobre el proceso de integración y de especial protagonismo del BCIE tras la reunión del Grupo Consultivo 4 en Madrid el 7 y 8 de marzo de 2001.

El proceso centroamericano de integración regional ocupó un lugar central en el Grupo Consultivo de Madrid y favoreció una amplia discusión sobre un interesante espectro de proyectos de carácter regional. Sin embargo, las expectativas generadas por el Grupo Consultivo se han convertido en una muestra más del constante desaprovechamiento de las potencialidades de la integración y de las dificultades que el proceso enfrenta.

La Conferencia reunió a algunas de las principales personalidades centroamericanas en torno a la reflexión sobre los 40 años de integración regional y de vida del $\mathrm{BCIE}$. La presencia en la conferencia de conocidos funcionarios, intelectuales y expertos en el tema como Gert Rosenthal, Eduardo Lizano, Haroldo Rodas, Fernando Mudarra y Ennio Rodríguez, permitió un debate de gran altura en el que se huyó de la retórica para centrar la discusión en algunos de los factores críticos del proceso. La especial relevancia de los conferenciantes permitió un debate rico, libre de la retórica de la integración por la integración. No es fácil disfrutar de un debate de estas características en torno al proceso de integración regional. La conferencia se celebró con la presencia de más de un centenar de invitados. Todas las sedes centroamericanas del $\mathrm{BClE}$ participaron en el evento mediante videoconferencia.

Pese a los síntomas de reanimación del proceso que se han percibido en los últimos años, la integración centroamericana sigue sin concretar avances definitivos. Las propuestas del Grupo Consultivo, después recogidas en

4 El Grupo Consultivo de Madrid de marzo de 2001 organizado por el Banco Interamericano de desarrollo (BID) y el gobierno español reunió a los gobiernos y agentes sociales de todos los países centroamericanos, los principales países donantes, las organizaciones internacionales presentes en Centroamérica, organizaciones no gubernamentales del Norte y otros miembros de la comunidad internacional. La reunión del grupo consultivo tuvo como eje la transformación y modernización de los países centroamericanos en clave regional y con especial énfasis en los esfuerzos de integración. Esta reunión daba seguimiento a la reunión del Grupo Consultivo de Estocolmo en mayo de 1999 que se centró en la transformación y reconstrucción de la región centroamericana tras el paso del Huracán Mitch. La reunión de Estocolmo definió la lucha contra la pobreza y la cooperación regional como principios de partida en la reducción de la vulnerabilidad y generó unas enormes expectativas en todo la región. NI Estocolmo ni Madrid han acabado respondiendo a ellas. 
buena medida por el Plan Puebla Panamá ; las propuestas de la UE y la AID en el conjunto de documentos de Centroamérica 2020; las propuestas de Harvard-INCAE (Instituto Centroamericano de Administración de Empresas); el interés de la sociedad civil por el proceso, han sido elementos muy positivos para el debate y un impulso al proceso.

Sin embargo, ninguna de estas opciones hallegado a cuajar y la integración no logra superar su condición de segunda opción marginada ante las negociaciones del ALCA (Área de libre comercio de las Américas) 0 ante cualquiera de las estrategias nacionales que anteponen frecuentemente acuerdos comerciales unilaterales a los acuerdos centroamericanos.

$\mathrm{Ni}$ siquiera las posibilidades de avance que sugieren las negociaciones para el establecimiento definitivo de la Unión aduanera en la región, o particularmente entre Guatemala y El Salvador ${ }^{6}$, parecen ofrecer excesivas garantías.

Precisamente, la consolidación de una unión aduanera plena y con reglas del juego claras es uno de los factores clave para el éxito de la integración. Su convivencia con acuerdos comerciales externos que no vulneren los acuer-

5 El gobierno mexicano del presidente Fox ha presentado una propuesta de cooperación económica y puesta en marcha de proyectos de infraestructuras que enlacen el sur de México (desde Puebla) con Centroamérica. El Plan Puebla Panamá ocupa en la actualidad el centro de los debates sobre el proceso centroamericano de integración. Su aparición ha supuesto la marginación de las propuestas que el INCAE había realizado con el equipo de la Universidad de Harvard dirigido por Michael Porter o de las propuestas derivadas del documento Centroamérica 2020 financiado por la AID y la UE pero con una visión más cercana a las tesis europeas. En estetrabajo ha tenido especial protagonismo Victor Bulmer-Thomas, uno delos principales expertos europeos sobre la integración centroamericana.

Los últimos años de la integración centroamericana se han caracterizado por la aparición de varias y sugestivas propuestas que se van reemplazando en el centro del debate sin oportunidad de análisis profundos. La profusión de documentos, estudios y propuestas acaba impidiendo un debate definitivo sobre las prioridades del proceso y la confirmación de las voluntades políticas de apoyarlo.

6 Ante la parálisis sufrida por el proceso centroamericano de integración regional tras el anuncio del ALCA en 1994, Guatemala y El Salvador iniciaron pocos años después una negociación bilateral para avanzar en el establecimiento de la Unión Aduanera. Esta decisión de los dos países de la región con más capacidad de impulsar el proceso y con más intereses comerciales en la unión aduanera, parecía una oportunidad de desbloquear el establecimiento definitivo de la Unión Aduanera. El retraso de las negociaciones y el conflicto generado entre Honduras y Nicaragua en el año 2000 por cuestiones limítrofes, acabó incorporando a estos dos países a la negociación. Como ha ocurrido en otras ocasiones, la negociación a 4 ofrece menos posibilidades de éxito. 
dos centroamericanos es un segundo factor crítico del proyecto.

En tercer lugar, el fortalecimiento de las instituciones y del marco general de la institucionalidad centroamericana es también un requisito fundamental para que la integración pueda desarrollar sus potencialidades. Para ello, el asunto de la financiación de las instituciones y sus acciones se convierte en tema prioritario. La capacidad para desarrollar políticas comunes que proporcionaría la existencia de mecanismos automáticos de financiación, podría permitir que el proceso mostrara por fin sus virtudes. Y, de paso, legitimaría a las propias instituciones ante las sociedades centroamericanas.

Y finalmente, en el desarrollo de estas políticas comunes el BCIE podría desempeñar un papel protagonista y cualitativamente decisivo. La autonomía financiera y política del $\mathrm{BCIE}^{7}$ con respecto al resto de la institucionalidad regional; su disponibilidad de recursos; y la experiencia aquilatada desde 1960, le conceden unas características que pueden hacer pensar en el $\mathrm{BCIE}$ como el eje del desarrollo equilibrado de los países centroamericanos en el marco de la integración.

Tanto las conferencias de los distintos ponentes como la mesa redonda fueron muy sugerentes al proponer nuevas funciones y prioridades para la propia integración regional y para el futuro del BCIE. La abundancia de propuestas de inversión en infraestructuras ha puesto de nuevo sobre la mesa el tema pero cabría pensar también en convertir al BCIE en un eje de la lucha contra la pobreza en todos los países a través de programas regionales.

En definitiva, se trató de una interesante y sugestiva celebración de 40 años de esfuerzo integrador. A continuación, se presenta una síntesis de las principales ideas expuestas por los conferenciantes.

\section{Integración y desarrollo en Centroamérica}

El actual Presidente del Banco Central de Costa Rica, Eduardo Lizano, presentó una visión del proceso marcada por lo que Gert Rosenthal Ilamaría después un sereno pesimismo. Su exposición consistió en una revisión del entorno, las condiciones y los retos a los que se enfrenta la región.

7 El BCIE dispone del capital social aportado por los socios centroamericanos y por los socios extrarregionales (Taiwan, Argentina, México y Colombia). El gobierno español está estudiando la posibilidad de incorporarse como socio extrarregional. El BCIE disfruta además de relaciones privilegiadas con el BID.

\section{RFS}


Según Lizano, Centroamérica tiene cada vez menos impacto en un entorno que sigue marcado por el consenso de Washington. Pero éste se encuentra en pleno proceso de revisión e incorporación de asuntos tradicionalmente poco atendidos por el banco Mundial como la lucha contra la pobreza y la protección medioambiental.

En esas circunstancias, según Lizano, la región se enfrenta a dos grandes retos: poner cada casa en orden y reorganizar el esquema de integración regional.

Es preciso poner la casa en orden y seguir construyendo el estado de derecho en cada país, vigilando los equilibrios macroeconómicos, abordando reformas tributarias o del sistema financiero y reorientando su gasto público. Lizano propuso para ello una sugerente fórmula 6/4/6/4 definida por un gasto en educación del $6 \%$ del presupuesto nacional, un gasto equivalente al $4 \%$ del PNB para el decil de renta más bajo de la población, garantizando un crecimiento del PIB del $6 \%$ anual y una inflación no superior al $4 \%$.

La reordenación del esquema de integración gira según Lizano en torno a varios elementos como la creación de mecanismos eficaces para la resolución de conflictos y los avances hacia la construcción de un espacio económico regional en torno a los sectores agrarios y de servicios, aprovechando los acuerdos sobre arancel externo común y procedimientos aduaneros.

Pese a ello, Lizano dibujó una integración que tiende a diluirse en una zona de libre comercio y que sufre las consecuencias de las posiciones individuales adoptadas por los países centroamericanos en las negociaciones externas. La ausencia de posiciones conjuntas pero flexibles en foros como el ALCA o la OMC (Organización Mundial de Comercio) perjudican al proceso tanto como la tendencia de los mandatarios de la región a tomar múltiples decisiones sin después ponerlas en marcha. Este panorama llevó a Lizano a preguntarse si la integración centroamericana se diluirá en el ALCA y si el eje Guatemala- El Salvador no podría convertirse en la línea de más fácil avance del proceso

\section{La globalización y el nuevo regionalismo}

La conferencia de Ennio Rodríguez, economista principal de la división de integración, comercio y asuntos hemisféricos del departamento de Integración y programas regionales del Banco Interamericano de Desarrollo, se 
centro en los elementos que definen el nuevo regionalismo en este entorno de globalización.

Entre los factores que marcan la globalización, Ennio Rodríguez destacó la búsqueda de una mayor eficiencia a partir de una creciente división del trabajo y de la nueva ingeniería industrial; la transmisión de efectos expansivos y recesivos; la distribución de los beneficios; la gobernabilidad; la revolución de las expectativas; los límites de la política económica; la conciencia de errores y efectos negativos de carácter global en temas financieros y ambientales; la necesidad de regular los conflictos; y los desafíos que impone el desarrollo de la democracia.

En este entorno, según Rodríguez, el nuevo regionalismo está marcado por una lógica de competitividad global que demanda la conexión con los centros norteamericanos de producción, distribución y consumo; la reducción delos costes de transacción y la armonización de regulaciones para crear un enfoque radial o de sistema.

Con esta concepción, Rodríguez señaló los siguientes factores de aprovechamiento para Centroamérica: la vecindad a los centros de consumo, la máxima división del trabajo, el desarrollo de infraestructuras de comunicación y transportes; la atención a los equilibrios macroeconómicos y el fomento de climas atractivos para la inversión externa y la apuesta por el desarrollo humano y sostenible.

\section{Lecciones del proceso europeo de integración en Centroamérica}

Fernando Mudarra, coordinador de la AECI (Agencia Española de Cooperación Internacional) en Honduras, expuso los factores de éxito del proceso europeo de integración regional que pueden ser de interés para la región centroamericana. Las tesis principales de esta conferencia ya fueron expuestas por Fernando Mudarra en su comparecencia en el Grupo Consultivo de Madrid junto a Pedro Caldentey, profesor de ETEA ${ }^{8}$ (institución universitaria española) y experto en integración europea y latinoamericana.

8 Estas tesis parten del trabajo publicado por José Juan Romero, Ma Luz Ortega y Pedro Caldentey, profesores del área de economía aplicada de ETEA, "Siete claves para comprender el proceso de construcción europea", en los Cuadernos socioeconómicos del Banco Centroamericano de Integración Económica (BCIE), no 26, diciembre 2000. Tegucigalpa (Honduras), edita Departamento de planificación y presupuesto del BCIE. 
La experiencia europea ha dejado, según Fernando Mudarra, tres lecciones básicas: el pragmatismo, la juridicidad y el gradualismo del proceso.

La Unión Europea según la exposición del autor ha ido transitando progresiva y gradualmente por distintas etapas del proceso de integración a partir de fundamentos históricos relacionados con la ll Guerra Mundial que no conviene olvidar para analizar el proceso y que explicaba ya en 1950 Robert Schuman en la trascendental declaración a la que dio nombre:

\begin{abstract}
"Para que la paz tenga realmente alguna oportunidad, primero necesitamos una Europa. Casi cinco años después de la rendición incondicional de Alemania, Francia emprende el paso decisivo en la construcción europea e invita a Alemania a participar en ésta. Esto debe transformar por completo las condiciones en Europa. Dicha transformación hace posible una nueva acción conjunta que hasta ahora ha sido imposible" (Declaración Schuman, 9 de mayo de 1950).
\end{abstract}

El tránsito progresivo de la UE desde la creación de la Comunidad Europea del Carbón y del Acero (CECA) en 1951 hasta la Unión Monetaria, pasando por la Unión aduanera del Tratado de Roma o el Mercado Único del Acta Única Europea demuestra que el gradualismo del proceso es un factor decisivo de éxito para el proceso de integración.

Lo económico ha sido determinante en este caminar lento hacia la profundización de la integración. Así lo explicaba otro de los grandes nombres de la UE, J ean Monnet:

"Logremos primero acuerdos económicos que permitan hacer ver a los europeos las ventajas de la unión; así iremos creando las condiciones que posibiliten afrontar en el futuro la unidad política de Europa".

Y lo institucional ha sido, según la explicación de Fernando Mudarra, también otro factor decisivo. La existencia de unas reglas del juego muy claras que permiten asegurar el cumplimiento de los acuerdos firmados en el marco de un entramado institucional que reparte competencias en un juego de equilibrios entre los poderes nacionales y comunitarios

Fernando Mudarra se refirió también a los mecanismos presupuestarios y de solidaridad que constituyen una de los principales elementos definitorios del modelo europeo de integración regional. Glosó también el debate sobre los retos de la Unión europea que se enfrenta hoy a una ampliación geográfica que va a exigir cambios muy importantes en instituciones y políticas. Se enfrenta también a la profundización de algunas de sus políticas. 


\section{Tratados preferenciales de comercio y la integración económica centro- americana}

Haroldo Rodas, Secretario general de la SIECA (Secretaria de la Integración Económica Centroamericana) expuso los avances que se han producido en el proceso de integración en el período más reciente y valoró sus perspectivas.

Según Rodas, la negociación de los mecanismos de solución de diferencias es uno de los asuntos sensibles que pueden ayudar a reducir el tamaño de alguno de los obstáculos que frenan el proceso.

Otros asuntos de mayor magnitud como la negociación del ALCA plantean también algunas dificultades al proceso. El Secretario General de la SIECA hizo un llamamiento a la necesidad de que Centroamérica actúe en el marco de los acuerdos regionales de unión aduanera. Estos acuerdos ofrecen un entorno de comercio y desarrollo muy amplio ya que sólo seis productos están excluidos del libre comercio.

No obstante, según Haroldo Rodas, es difícil que los países de la región puedan adoptar acuerdos so bre su arancel externo si perforan continuamente el MCCA con negociaciones unilaterales.

Otros avances permiten pensar en un entorno favorecedor de la integración pero quizás el más sugerente de los que discute hoy la región es la negociación de un mecanismo de recaudación conjunta que pueda proporcionar financiación a las posibles políticas comunitarias La SIECA y otras instituciones regionales están estudiando la creación de un fondo regional financiado a medias entre los recursos aduaneros y la cooperación internacional. Los criterios de reparto serían diversos e irían desde criterios geográficos por país o municipios hasta determinados sectores sociales y económicos

Pese a los avances reseñados, Haroldo Rodas no dejó de señalar el desafío que supone el ALCA, un escenario ante el que los países centroamericanos deben prepararse. 


\section{Centroamérica ante la globalización e integración}

La muy sugestiva intervención de Gert Rosenthal ${ }^{9}$, ex-Secretario General de la CEPAL (Comisión económica para América Latina y el Caribe de las Naciones Unidas) y actualmente Representante Permanente de Guatemala en Naciones Unidas, se quiso situar entre lo que el conferenciante denominó el sereno optimismo de Haroldo Rodas y el sereno pesimismo de Eduardo Lizano.

Como ha venido expresando desde hace años, Rosenthal partió del desaprovechamiento del potencial de la integración. La última oportunidad fue el Grupo consultivo de Madrid del que el conferenciante afirmó haber salido con una doble sensación. Por una parte, una sensación de confianza por el interés y optimismo por la idea de una Centroamérica integrada y por la imagen de una cooperación internacional centrada en el regional. Por otra parte, con la triste sensación de que no había ni pasión ni compromisos en torno a este interés por lo regional.

Una vez más, la retórica de la integración parece perder la batalla con la realidad y aunque se mantienen los profundos lazos de interdependencia que tiene la región, no parecen ser suficientes para resolver sus problemas y por ello se ha venido extendiendo una estrategia de sálvese quien pueda.

\section{Cuatro fenómenos explican la distancia entre la teoría y la práctica}

En primer lugar, la región ha subestimado las dificultades de la transición política. En segundo lugar, se subestimaron también los costos de una transición económica encaminada a la adaptación a la economía globalizada. En tercer lugar, no ha habido apoyo político suficiente para las instituciones de la integración centroamericana. Y en cuarto lugar, señaló Rosenthal, la globalización ha generado más impulsos desagregadores que integradores, fomentando una cierta rivalidad entre los países socios por los mercados exteriores.

¿Cómo capitalizar el potencial de la integración en estos tiempos de globalización? Rosenthal propuso seis áreas de colaboración entre las políticas nacionales y una visión regional:

9 Publicada íntegramente en el número 223 de la Revista de Fomento Social. 
- Profundizar las democracias emergentes en todos los países centroamericanos.

- Fortalecer las instituciones y organizaciones de cada país, públicas y privadas.

- Asumir el imperativo de recortar el rezago tecnológico de las empresas de la región.

- Invertir en los recursos humanos para enfrentarse al desafío de la desigualdad y la pobreza.

- Mejorar la calidad de la gestión macroeconómica para asegurar estabilidad, crecimiento y equidad.

- Desarrollar los principios de Guácimo y ALIDES (Alianza para el desarrollo sostenible) para garantizar modelos de desarrollo sostenibles.

Para finalizar, Rosenthal remarcó la necesidad de aprovechar las potencialidades de la integración aunque en el coloquio mostró su escepticismo ante la posibilidad de resolver problemas como la falta de cultura de la integración, la compatibilidad de los elementos de política económica con los de política exterior y la falta de entendimiento que en ocasiones se produce entre cancillerías y responsables de economía.

También, y durante el coloquio, se abordó el importante asunto de la institucionalidad regional. Gert Rosenthal sugirió trabajar para definir quién asume qué competencias, dotar a las instituciones de recursos y destinar a ellas los mejores recursos humanos.

\section{Presentación del libro “El desarrollo económico de Centroamérica en el marco de la integración regional"}

Durante la conferencia, se presentó el libro "El desarrollo económico de Centroamérica en el marco de la integración regional", editado por el BCIE y obra de Pedro Caldentey del Pozo, profesor de ETEA, institución universitaria de Córdoba (España) y experto en integración centroamericana.

El presidente del $\mathrm{BCIE}, \mathrm{D}$. Pablo Schneider presentó el libro destacando su aportación al conocimiento del proceso de integración, merced al exhaustivo recorrido por el proceso de integración. El autor agradeció la presentación del libro y la decisión de publicarlo al Presidente del BCIE y a Carlos Imendia, Director de Planificación y Presupuestos del BCIE. 
Finalmente se celebró una mesa redonda sobre El papel del BCIE en la integración económica y el desarrollo sostenible de Centroamérica presidida por Fernando García, Vicepresidente del BCIE, y con la participación detodos los ponentes a los que se sumó Pedro Caldentey. Con ella se cerró la conferencia.

\section{Algunas conclusiones}

La integración centroamericana avanza entre grandes dificultades desde sus comienzos y sólo sobrevive porque se impone la lógica de unir esfuerzos comunes entre países de reducido tamaño en este entorno de globalización.

Es frecuente que en los eventos relacionados con la integración centroamericana se recurra a la tradición integracionista de la región remontándose a la Patria Centroamericana de antes de la independencia y de sus primeros años, o recordando los numerosos esfuerzos de integración política impulsados desde 1821 por próceres y gobiernos. Este hábito, pese al interés de esos antecedentes, no ha beneficiado en exceso al proceso ya que los Ilamamientos realizados se perciben como una retórica alejada de la realidad. Casi como una excusa de los gobernantes nacionales para hablar de integración sin realizar compromisos concretos de avance.

La Conferencia regional que reseñamos se alejó de la retórica de la integración centroamericana y aprovecho la oportunidad de reunir a ponentes de gran interés para sostener un debate bastante profundo sobre la factibilidad del proceso y sobre sus principales obstáculos. Puso también en evidencia la diferente percepción que se tiene del proceso desde cada país o desde cada institución.

Entre los asuntos comentados, destaca por ejemplo, la necesidad de retomar la reforma institucional. La Cumbre de presidentes de Panamá en julio de 1997 acordó poner en marcha una racionalización de la institucionalidad centroamericana a partir de la propuesta del BID y la CEPAL, con el objetivo de simplificarla y de hacerla más eficaz ${ }^{10}$. La reforma planteada

10 El marco institucional de la integración centroamericana se compone de más de 40 instituciones con recursos, sedes y poder político muy dispersos. La reforma institucional propone como uno de sus ejes la simplificación de este esquema y la agrupación de Secretarías técnicas e institutos en torno a la Secretaría general del Sistema, que con sede en San Salvador, podría ejercer un papel similar al de la Comisión Europea en la UE. 
suscitó gran polémica entre al gunas de las instituciones más afectadas como el Parlamento Centroamericano, la Corte Centroamericana de Justicia y la SIECA.

Más allá de algunas medidas discutibles, es evidente la necesidad de ejecutar esa reforma y de garantizar que la institucionalidad regional tiene capacidad de negociación política ante los gobiernos nacionales. Es preciso garantizar también la autonomía presupuestaria y la dotación de recursos para que las instituciones puedan desarrollar sus políticas sin excesiva dependencia de las voluntades de colaboración de cada gobierno. En esta línea, son muy interesantes las discusiones sobre el uso de parte de la recaudación arancelaria -en un escenario de unión aduanera que funcionapara sufragar la factura institucional.

Otro de los aspectos críticos del proceso es la necesidad de ofrecer avances en el proceso y resultados tangibles derivados de éste a las sociedades centroamericanas. La profusión de acuerdos firmados y compromisos no cumplidos afecta gravemente a la legitimidad del proceso. La experiencia europea podría ser retomada en este caso para empezar por los asuntos de ámbito económico que tienen un resultado más fácilmente visible. En concreto, el establecimiento de la Unión Aduanera podría generar transformaciones importantes en pocos años si se le diera la oportunidad de demostrarlo. Recuperar el impulso de la negociación entre El Salvador y Guatemala, podría ser la clave para alcanzar este objetivo. El papel de la SIECA ante esta negociación debe ser reforzado.

Del mismo modo, y como señalaron algunos ponentes en la conferencia, el desafío del ALCA y la compatibilidad de los acuerdos comerciales de los socios centroamericanos, se convierte en otro factor determinante para el proceso. Pese a los intentos de la SIECA por crear un marco de negociaciones donde se pueda hablar de un arancel externo común pero hacer negociaciones comerciales unilaterales y luego buscar mecanismos que compatibilicen ambas cosas, el entorno es tremendamente confuso. Resulta francamente difícil saber cuál es el estado actual de las negociaciones comerciales de cada uno de los socios y de todos en conjunto.

Los países centroamericanos parecen despreciar las opciones que ofrece un esfuerzo común en la negociación de acuerdos comerciales. La presencia de una Centroamérica con un criterio común ante las negociaciones del ALCA ofrecería perspectivas optimistas. Lamentablemente, la realidad se aleja mucho de ello y, si no se producen cambios sustanciales, los acuerdos 
regionales centroamericanos están comprometidos por los resultados del ALCA.

Finalmente, una de las principales cuestiones que plantea este seminario es la necesidad de reflexionar y estudiar el rol del BCIE en la integración regional. Tras cuarenta años de experiencia, el Banco se encuentra en una posición difícilmente mejorable para convertirse en protagonista del proceso. Su autonomía financiera, la presencia de socios extrarregionales y la cercanía con el BID, y la experiencia de desarrollo de programas regionales, hacen del BCIE una de las pocas instituciones con capacidad de ofrecer resultados tangibles.

Ha sido frecuente oír en el ámbito de la integración centroamericana que el BCIE no ha cumplido su función de apoyo al proceso a favor de sus tareas de banco de desarrollo de la región o de determinadas actividades de las décadas más recientes cuestionadas por su carácter comercial.

Pese a estas críticas, la historia del BCIE muestra grandes potencialidades y muchos buenos resultados. La inversión en infraestructuras en la década de los sesenta es un buen ejemplo de ello.

Es probable que un análisis pormenorizado de los proyectos gestionados en estos 40 años y de la distribución por países de estos fondos, nos proporcionaran una visión muy sugerente sobre las posibilidades del BCIE como eje del desarrollo equilibrado en la integración centroamericana.

El catálogo de proyectos presentados por las instituciones regionales al Grupo Consultivo y que luego ha retomado en buena parte el Plan Puebla Panamá, pone de nuevo en evidencia que el $\mathrm{BCIE}$ puede constituirse en el motor y gestor de una política similar en algunos aspectos a la política estructural o de desarrollo regional y cohesión de la Unión Europea.

Esta hipótesis, que ya se plantea sin suspicacias en el entorno de los gobiernos y las instituciones regionales, permitiría diseñar una política basada en ciertos grandes ejes (luchas contra la pobreza, PYME, infraestructuras de transporte, etcétera) y en función de criterios de reparto basados no tanto en los países como en los colectivos sociales elegibles y en divisiones administrativas inferiores a la nacional para evitar agravios en el reparto de los fondos.

No es difícil imaginar que una política de desarrollo equilibrado y cohesión regional, con resultados positivos en la lucha contra la pobreza y en la 
modernización económica, atrajera el interés de la comunidad internacional y sus fondos.

¿Por qué no enfrentar al BCIE a este desafío? ¿Por qué no convertirlo en el eje de algunas de las políticas comunes de la integración centroamericana? 